\title{
On Certain Equations to the Cubic Surface, with Extensions to Higher Space
}

\author{
By W. Saddler (Christ Church, New Zealand). \\ (Received 18th March, 1933. Read 3rd November, 1933.)
}

The Cubic Surface, as is well known, can be formulated as the locus of a point which, when joined to six given lines-one of which cuts the other five-forms planes enveloping a quadric cone. ${ }^{1}$ It might be of some interest to show how such a definition leads to one or two of the better known forms of the equation to the surface. The method of approach is by means of the Clebsch Transformation Principle in Geometry and the use of general coordinates. In particular, compound symbols and bracket factors, as developed by H. W. Turnbull ${ }^{2}$ in his works on Geometry and Invariant Algebra, have been largely used.

In section $2(12)$ a form is obtained analogous to the type

$$
\left|\begin{array}{lll}
u & v & w \\
u^{\prime} & v^{\prime} & w^{\prime} \\
u^{\prime \prime} & v^{\prime \prime} & w^{\prime \prime}
\end{array}\right|
$$

where $u, v \ldots$ are planes and in $\S 2(14)$ a symmetrical type is found, which again leads (in $\S 3,2$ ) to the form $u v w=u^{\prime} v^{\prime} w^{\prime}$, besides admitting of extension to higher dimensions, though the manifolds in this case are not general. The case in five-fold space is a direct. analytical extension of that in three dimensions.

\section{$\S 1$.}

Consider briefly the underlying theory involved in the Clebsch Principle. We represent a point in three dimensional space by $\left(y_{1}, y_{2}, y_{3}, y_{4}\right)$, or by, $u_{y}=u_{1} y_{1}+u_{2} y_{2}+u_{3} y_{3}+u_{4} y_{4}$, or simply by $y$. Again a line through the two points $a, a^{\prime}$, with coordinates $\left(a a^{\prime}\right)_{i j}=\left(a_{i} a_{j}^{\prime}-a_{i}^{\prime} a_{j}\right)$, where $i, j=1,2,3,4$, is represented by $\left(a a^{\prime}\right)$, or by a compound symbol $A$. Thus a plane through the three points $y, z, t$, has the equation $(x y z t)=0$, a determinant of the fourth order.

${ }^{1}$ H. F. Baker, Principles of Geometry (1923) 3, 168.

$2 \mathrm{H}$. W. Turnbull, Determinants, Matrices and Invariants (1928), 212, 287. 
The point $\lambda a+\mu a^{\prime}$ of the line $\left(a a^{\prime}\right)$ lies in this plane when,

$$
\lambda(a y z t)+\mu\left(a^{\prime} y z t\right)=0
$$

and thus the point of intersection of the line and plane is given by

$$
u_{a^{\prime}}(a y z t)-u_{a}\left(a^{\prime} y z t\right) \equiv \Omega\left(a a^{\prime}\right) . u_{a^{\prime}}(a y z t),
$$

where $\Omega\left(a a^{\prime}\right)$ denotes the determinantal permutation of the letters indicated. By a fundamental identity an alternative form to this is

$$
u_{y}\left(a a^{\prime} z t\right)+u_{z}\left(a a^{\prime} t y\right)+u_{t}\left(a a^{\prime} y z\right) \equiv \Omega(y z t) \cdot u_{y}\left(a a^{\prime} z t\right) .
$$

This expresses the point, in the plane of the triangle $y, z, t$, by

where $\lambda_{1}=\left(a a^{\prime} z t\right)$, etc.

$$
\lambda_{1} y+\lambda_{2} z+\lambda_{3} t
$$

Such scalar quantities $\lambda_{i}$ may be regarded as ternary coordinates of a point $\lambda$ in the plane of this triangle. If $\left(a a^{\prime}\right),\left(b b^{\prime}\right),\left(c c^{\prime}\right)$ are three skew lines cutting this plane in points $\lambda, \mu, \nu$, then the expression

$$
(\lambda \mu \nu)=\Sigma \pm \lambda_{1} \mu_{2} \nu_{3}=\left|\begin{array}{ccc}
\left(a a^{\prime} z t\right) & \left(a a^{\prime} t y\right) & \left(a a^{\prime} y z\right) \\
\left(b b^{\prime} z t\right) & \left(b b^{\prime} t y\right) & \left(b b^{\prime} y z\right) \\
\left(c c^{\prime} z t\right) & \left(c c^{\prime} t y\right) & \left(c c^{\prime} y z\right)
\end{array}\right|
$$

is a projective invariant. It connects the ternary bracket factor $(\lambda \mu \nu)$ with a quaternary invariant. This latter is also equal to

$$
\Omega\left(a a^{\prime}, b b^{\prime}\right) \cdot\left(c c^{\prime} a^{\prime} b^{\prime}\right)(a y z t)(b y z t)
$$

where the $\Omega$ operator indicates a determinantal permutation of $a$ with $a^{\prime}$, and $b$ with $b^{\prime}$, and involves four terms. Lastly, writing $u$ for $(y z t)$, this becomes

$$
(u C A B u) \equiv \Omega\left(a a^{\prime}, b b^{\prime}\right) \cdot\left(c c^{\prime} a^{\prime} b^{\prime}\right) u_{a} u_{b} .
$$

Thus the ternary bracket factor of the type $(\lambda \mu \nu)$ admits of a transference to the compound quaternary factor of the type (4), where $A, B, C$ are the coordinates of the three lines. It gives the well known result, when equated to zero, that the envelope of a plane cutting three lines in three collinear points is a quadric surface of which the three given lines are generating lines.

Similarly for four dimensional space, a bracket factor of the type $(\lambda \mu \nu \rho)$ where as before the $\lambda, \mu, \nu, \rho$ are associated with the points in which the lines $\left(a a^{\prime}\right),\left(b b^{\prime}\right),\left(c c^{\prime}\right),\left(d d^{\prime}\right)$ meet the prime (three-fold) $u$, through the points $x, y, z, t$ goes over into

$$
\Omega\left(a a^{\prime}, b b^{\prime}, c c^{\prime}\right) .\left(d d^{\prime} a b c\right) u_{a^{\prime}} u_{b^{\prime}} u_{c^{\prime}},
$$

a determinantal expression involving permutations of $a, a^{\prime} ; b, b^{\prime}$; $c, c^{\prime}$, independently. 
Instead of representing the line as joining the two points $a, a^{\prime}$, with coordinates $\left(a a^{\prime}\right)$, let us regard it as the intersection of two planes, $a_{x} \equiv a_{1} x_{1}+a_{2} x_{2}+a_{3} x_{3}+a_{4} x_{4}=0$ and $\beta_{x}=0$, and thus, but for a constant factor, let

$$
\left(a a^{\prime}\right)_{i j}=\left(\alpha \alpha^{\prime}\right)_{k l,}, \quad(i, j ; k, l)=(1,2,3,4) .
$$

Then $(u A B C u)$ will go over into

$$
(u P Q R u)=\Omega\left(a \alpha^{\prime}\right) .\left(\beta \beta^{\prime} a u\right)\left(\gamma \gamma^{\prime} \alpha^{\prime} u\right)
$$

where now $u, a, \beta, \gamma$ are cogredient symbols.

Similarly, for the four dimensional case, in which the lines will be represented by the intersection of three primes (three-folds), so that

then,

$$
\left(a a^{\prime}\right)_{i j}=\left(a \alpha^{\prime} \alpha^{\prime \prime}\right)_{k l m},
$$

$$
\Omega\left(a a^{\prime}, b b^{\prime}, c c^{\prime}\right) \cdot\left(d d^{\prime} a b c\right) u_{a^{\prime}} u_{b^{\prime}} u_{c^{\prime}},
$$

will, but for a constant factor, be equal to

$$
\begin{aligned}
& \Omega\left(\beta \beta^{\prime} \beta^{\prime \prime}\right) \cdot\left(\alpha \alpha^{\prime} \alpha^{\prime \prime} \beta u\right)\left(\gamma \gamma^{\prime} \gamma^{\prime \prime} \beta^{\prime} u\right)\left(\delta \delta^{\prime} \delta^{\prime \prime} \beta^{\prime \prime} u\right) \\
= & \Omega\left(\beta \beta^{\prime} \beta^{\prime \prime}\right) \cdot\left(A_{3} \beta u\right)\left(C_{3} \beta^{\prime} u\right)\left(D_{3} \beta^{\prime \prime} u\right) .
\end{aligned}
$$

This result, when equated to zero, implies that the envelope of a three-fold cutting four lines in four points, which lie in a plane, is a cubic manifold.

The dual theorems are established in a similar manner: the interchange of point and prime coordinates gives the corresponding algebraical results. Thus in five-fold space, the locus

$$
\Omega\left(e e^{\prime} e^{\prime \prime} e^{\prime \prime \prime}\right) \cdot\left(A_{4} e x\right)\left(B_{4} e^{\prime} x\right)\left(C_{4} e^{\prime \prime} x\right)\left(D_{4} e^{\prime \prime \prime} x\right)=0
$$

is a quartic manifold locus of a point such that the five four-folds obtained by joining it to the five three-folds $A_{4}, B_{4}, C_{4}, D_{4}, E_{4}$, have a line in common. Here

$$
E_{4}=\left(e e^{\prime} e^{\prime \prime} e^{\prime \prime \prime}\right)
$$

the $e$ symbols being cogredient with the point symbols, $x$. This locus is a natural extension of the quadric in [3],

$$
(x A B C x)=\Omega\left(c c^{\prime}\right) \cdot(c A x)\left(c^{\prime} B x\right)=0 .
$$

It is clear that the five three-folds lie wholly on the manifold, just as the lines $A, B, C$, are generators of the quadric surface in [3]. 


\section{$\S 2$.}

Consider now six lines in three dimensional space represented by $A, B, C, D, E, F$ where $A=\left(a a^{\prime}\right)$, and consider the envelope of a plane which cuts these six lines in points lying on a conic. Now the points $\lambda, \mu, \nu, \rho, \sigma, \tau \S 1(2)$ will lie on a conic if

$$
(\lambda \nu \sigma)(\tau \lambda \mu)(\mu \nu \rho)(\rho \sigma \tau)-(\mu \rho \tau)(\lambda \mu \nu)(\nu \rho \sigma)(\sigma \tau \lambda)=0^{1} .
$$

Hence the envelope of the plane cutting the six lines in points on a conic is the surface

$$
\begin{aligned}
& (u A C E u)(u F A B u)(u B C D u)(u D E F u) \\
& \quad-(u B D F u)(u A B C u)(u C D E u)(u E F A u)=0 .
\end{aligned}
$$

It is more useful for our present purpose to consider the dual of this result: namely the locus of a point such that the planes joining it to six lines touch a quadric cone. This locus is of the eighth degree (since $x$ enters eight times into each term) and has the equation

where

$$
\begin{aligned}
& (x A C E x)(x F A B x)(x B C D x)(x D E F x) \\
& -(x B D F x)(x A B C x)(x C D E x)(x E F A x)=0,
\end{aligned}
$$

$$
(x A B C x)=\Omega\left(b b^{\prime}\right) \cdot\left(a a^{\prime} b^{\prime} x\right)\left(b c c^{\prime} x\right) .
$$

In the special case where the line $F$ meets the other five lines, it will now be shewn that the locus reduces to a cubic surface.

For certain values of the scalar parameters $\alpha, \beta, \gamma, \delta, \epsilon, \mu, \nu, \ldots \tau^{\prime}$,

Hence

$$
\begin{aligned}
& a+\alpha a^{\prime} \equiv \mu f+\mu^{\prime} f^{\prime} \\
& b+\beta b^{\prime} \equiv \nu f+\nu^{\prime} f^{\prime} \\
& c+\gamma c^{\prime} \equiv \rho f+\rho^{\prime} f^{\prime} \\
& d+\delta d^{\prime} \equiv \sigma f+\sigma^{\prime} f^{\prime} \\
& e+\epsilon e^{\prime} \equiv \tau f+\tau^{\prime} f^{\prime} .
\end{aligned}
$$

$(x F A B x)=\Omega\left(a a^{\prime}\right) \cdot\left(f f^{\prime} a^{\prime} x\right)\left(a b b^{\prime} x\right)=\left(\mu \nu^{\prime}-\mu^{\prime} \nu\right)\left(f f^{\prime} a^{\prime} x\right)\left(f f^{\prime} b^{\prime} x\right) ;$

and similarly for the other bracket factors involving $F$.

The equation (2), thus reduces to the form

$$
\begin{aligned}
& \left(\mu \nu^{\prime}-\mu^{\prime} \nu\right)\left(\sigma \tau^{\prime}-\sigma^{\prime} \tau\right)(x A C E x)(x B C D x) \\
& -\left(\nu \sigma^{\prime}-\nu^{\prime} \sigma\right)\left(\mu \tau^{\prime}-\mu^{\prime} \tau\right)(x A B C x)(x C D E x)=0 .
\end{aligned}
$$

1 Cf. Grace and Young, Algebra of Invariants, 280. 
But, by (3),

$$
\begin{aligned}
& a \beta\left(a a^{\prime} b b^{\prime}\right)=-\left(\mu \nu^{\prime}-\mu^{\prime} \nu\right)\left(f f^{\prime} a b\right) \\
& \delta \epsilon\left(d d^{\prime} e e^{\prime}\right)=-\left(\sigma \tau^{\prime}-\sigma^{\prime} \tau\right)\left(f f^{\prime} d e\right) \\
& a \epsilon\left(a a^{\prime} e e^{\prime}\right)=-\left(\mu \tau^{\prime}-\mu^{\prime} \tau\right)\left(f f^{\prime} a e\right) \\
& \beta \delta\left(b b^{\prime} d d^{\prime}\right)=-\left(\nu \sigma^{\prime}-\nu^{\prime} \sigma\right)\left(f f^{\prime} b d\right),
\end{aligned}
$$

and thus (4) becomes

$$
\begin{aligned}
& (x A C E x)(x B C D x)\left(f f^{\prime} a e\right)\left(f f^{\prime} b d\right)(A B)(D E) \\
& \quad-(x A B C x)(x C D L x)\left(f f^{\prime} a b\right)\left(f f^{\prime} d e\right)(A E)(B D)=0 .
\end{aligned}
$$

Denote the coordinates of the points $a+\alpha a^{\prime}, b+\beta b^{\prime}, c+\gamma c^{\prime}$, $d+\delta d^{\prime}, e+\epsilon e^{\prime}$, by $a^{\prime \prime}, b^{\prime \prime}, c^{\prime \prime}, d^{\prime \prime} ; e^{\prime \prime}$, and the equation retains the same form with $a^{\prime \prime}$ substituted for $a^{\prime}$ etc., while,

$$
\left(f f^{\prime} a^{\prime \prime}\right)_{i}=0=\left(f f^{\prime} b^{\prime \prime}\right)_{i}=\left(f f^{\prime} d^{\prime \prime}\right)_{i}=\left(f f^{\prime} e^{\prime \prime}\right)_{i}=\left(f f^{\prime} c^{\prime \prime}\right)_{i} .
$$

Again using (6)

$$
\begin{aligned}
& \left(f f^{\prime} b d\right)\left(a a^{\prime \prime} b b^{\prime \prime}\right)=\left(f f^{\prime} b a\right)\left(d a^{\prime \prime} b b^{\prime \prime}\right) \\
& \left(f f^{\prime} d e\right)\left(a a^{\prime \prime} e e^{\prime \prime}\right)=\left(f f^{\prime} a e\right)\left(d a^{\prime \prime} e e^{\prime \prime}\right),
\end{aligned}
$$

and hence $(5)$ reduces to

$$
\begin{aligned}
& (x A C E x)(x B C D x)\left(d d^{\prime \prime} e e^{\prime \prime}\right)\left(d a^{\prime \prime} b b^{\prime \prime}\right) \\
& +(x A B C x)(x C D E x)\left(b b^{\prime \prime} d d^{\prime \prime}\right)\left(d a^{\prime \prime} e e^{\prime \prime}\right)=\mathbf{0 .}
\end{aligned}
$$

There will be no confusion in what follows to take the points $a^{\prime \prime}, b^{\prime \prime} \ldots$ as $a^{\prime}, b^{\prime}, \ldots$, where these points are collinear on $F$. Expanding out these bracket factors $(x A C E x)$ and $(x A B C x)$ and using the symbolic identities, (8) becomes

Since

$$
\begin{aligned}
& \left(c c^{\prime} a^{\prime} x\right)\left(a e e^{\prime} x\right)(x B C D x)\left(b b^{\prime} d a^{\prime}\right)\left(d d^{\prime} e e^{\prime}\right) \\
+ & \left(c c^{\prime} a^{\prime} x\right)\left(a b b^{\prime} x\right)(x D C E x)\left(b b^{\prime} d d^{\prime}\right)\left(e e^{\prime} d a^{\prime}\right) \\
+ & \left(c c^{\prime} a x\right)\left(a^{\prime} b b^{\prime} x\right)\left(c c^{\prime} d^{\prime} x\right)\left(d e e^{\prime} x\right)\left(b b^{\prime} d d^{\prime}\right)\left(e e^{\prime} d a^{\prime}\right) \\
- & \left(c c^{\prime} a x\right)\left(a^{\prime} e e^{\prime} x\right)\left(c c^{\prime} d^{\prime} x\right)\left(b b^{\prime} d x\right)\left(b b^{\prime} d a^{\prime}\right)\left(d d^{\prime} e e^{\prime}\right)=0 .
\end{aligned}
$$

$$
\left(c c^{\prime} a^{\prime} x\right)\left(c c^{\prime} d^{\prime} a\right)=\left(c c^{\prime} d^{\prime} x\right)\left(c c^{\prime} a^{\prime} a\right),
$$

the non-vanishing factor $\left(c c^{\prime} a^{\prime} x\right)$ may be removed and the equation (9) reduces to that of the cubic surface,

$$
\begin{aligned}
& \left(a e e^{\prime} x\right)(x B C D x)\left(b b^{\prime} d a^{\prime}\right)\left(d d^{\prime} e e^{\prime}\right)\left(c c^{\prime} a a^{\prime}\right) \\
+ & \left(a b b^{\prime} x\right)(x D C E x)\left(b b^{\prime} d d^{\prime}\right)\left(e e^{\prime} d a^{\prime}\right)\left(c c^{\prime} a a^{\prime}\right) \\
- & \left(c c^{\prime} a x\right)\left(a^{\prime} b b^{\prime} x\right)\left(d e e^{\prime} x\right)\left(b b^{\prime} d d^{\prime}\right)\left(e e^{\prime} d a^{\prime}\right)\left(c c^{\prime} d^{\prime} a\right) \\
+ & \left(c c^{\prime} a x\right)\left(a^{\prime} e e^{\prime} x\right)\left(b b^{\prime} d x\right)\left(b b^{\prime} d a^{\prime}\right)\left(d d^{\prime} e e^{\prime}\right)\left(c c^{\prime} d^{\prime} a\right)=0 .
\end{aligned}
$$


Now,

$$
\begin{aligned}
(C a x) & \left(a^{\prime} E x\right)(B d x)\left(B d a^{\prime}\right)(D E)\left(C d^{\prime} a\right) \\
= & (C a x)\left(d^{\prime} E x\right)(B d x)\left(B d a^{\prime}\right)\left(d a^{\prime} E\right)\left(C d^{\prime} a\right) \\
= & (C a x)(x D B E x)\left(B d a^{\prime}\right)\left(E d a^{\prime}\right)\left(C d^{\prime} a\right) \\
& +(C a x)\left(B d^{\prime} x\right)(d E x)\left(B d a^{\prime}\right)\left(E d a^{\prime}\right)\left(C d^{\prime} a\right)
\end{aligned}
$$

Hence (10) reduces to

$$
\begin{gathered}
(a E x)(x B C D x)\left(B d a^{\prime}\right)(D E)(C A)+(a B x)(x D C E x)(B D)\left(E d a^{\prime}\right)(C A) \\
+(a C x)(x D B E x)\left(B d a^{\prime}\right)\left(E d a^{\prime}\right)\left(C d^{\prime} a\right)=0 .
\end{gathered}
$$

This can also be expressed as a determinant

$$
\left|\begin{array}{lll}
(a E x)(D E) & (a B x)(B D) & (a C x)\left(C a d^{\prime}\right) \\
(d E x)\left(E d a^{\prime}\right) & (d B x)\left(B d a^{\prime}\right) & (d C x)(C A) \\
\left(d^{\prime} E x\right)\left(E d a^{\prime}\right) & \left(d^{\prime} B x\right)\left(B d a^{\prime}\right) & \left(d^{\prime} C x\right)(C A)
\end{array}\right|=0 .
$$

Again (11) can further be simplified by using the three results

$$
\begin{aligned}
& (d C x)(x D B E x)=(d E x)(x D B C x)+(d B x)(x D C E x), \\
& \left(d^{\prime} C x\right)(x D B E x)=\left(d^{\prime} E x\right)(x D B C x)+\left(d^{\prime} B x\right)(x D C E x), \\
& (a E x)(D E)=(d E x)\left(E a d^{\prime}\right)+\left(d^{\prime} E x\right)(E d a) .
\end{aligned}
$$

The form for the surface becomes

$$
\begin{gathered}
(x C D E x)\left\{(d B x)\left(B d a^{\prime}\right)\left(E a d^{\prime}\right)(C A)+\left(d^{\prime} B x\right)\left(B d a^{\prime}\right)(E d a)(C A)\right. \\
\left.-(a B x)(B D)\left(E d a^{\prime}\right)(C A)\right\} \\
-(x B D E x)\left\{(C d x)\left(B d a^{\prime}\right)\left(E a d^{\prime}\right)(C A)+\left(d^{\prime} C x\right)\left(B d a^{\prime}\right)(E d a)(C A)\right. \\
\left.+(a C x)\left(B d a^{\prime}\right)\left(E d a^{\prime}\right)\left(C d^{\prime} a\right)\right\}=0,
\end{gathered}
$$

which, since

becomes

$$
\left(B d a^{\prime}\right)\left(B d^{\prime} a\right)=-(B D)(B A)
$$

$$
\begin{gathered}
(x B D E x)\left\{(C d x)(A B)(C A)\left(E a d^{\prime}\right)+\left(C d^{\prime} x\right)(A B)(C A)(E d a)\right. \\
\left.+(C a x)(A B)\left(C d^{\prime} a\right)\left(E d a^{\prime}\right)\right\} \\
-(x C D E x)\left\{(B d x)(A C)(A B)\left(E a d^{\prime}\right)+\left(B d^{\prime} x\right)(A C)(B A)(E d a)\right. \\
\left.+(B a x)(A C)\left(B d^{\prime} a\right)\left(E d a^{\prime}\right)\right\}=0 .
\end{gathered}
$$

It is to be observed that the first half of this expression is transformed to the second by the interchange of $B$ and $C$ : thus the surface can be written in the symmetrical form

$$
(x B D E x)(x C y)-(x C D E x)(x B y)=0
$$

where $y$ is a determined auxiliary point.

Here $(x B D E x)=0$ denotes a quadric in which $B, D, E$ are generators, and $(x C y)=0$ a plane through the point $y$ and the line $C$. 
From the form of (14), this can also be written as

$$
(x D B C x)(x E y)-(x E B C x)(x D y)=0 .
$$

But by manipulating the symbols in other orders or starting afresh from (8) the equation admits of different forms-all of the same type but with the auxiliary point $y$ changed to other points-for instance:

$$
(x A C D x)(x B z)-(x B C D x)(x A z)=0 .
$$

We shall see the meaning of these equations in the next section.

\section{$\S 3$.}

Consider now H. F. Baker's construction for the cubic surface. ${ }^{1}$ Take four given lines and a given point. Denote the point by $y$ and the lines by $\left(a a^{\prime}\right),\left(b b^{\prime}\right),\left(c c^{\prime}\right),\left(d d^{\prime}\right)$. Consider the lines grouped as two pairs $(A),(B) ;(C),(D)$.

A plane through $y$ cuts $(A),(B)$ in the points $P$ and $Q$ (say) and $(C),(D)$ in $R$ and $S:$ then the locus of the point $x$, where $P Q$ meets the line $R S$, for different planes through $y$ is the general cubic surface.

Its equation may be determined as follows. Thus $x$ and $\left(a a^{\prime}\right)$ determine a plane which cuts the line $\left(b b^{\prime}\right)$ in the point $Q$ whose coordinates are

$$
b\left(A b^{\prime} x\right)-b^{\prime}(A b x) .
$$

The join of $x Q$ will determine the unique line through $x$ to cut $A$ and $B$. Again $x$ and $\left(c c^{\prime}\right)$ determines a plane which cuts $\left(d d^{\prime}\right)$ in the point $S$, namely,

$$
d\left(C d^{\prime} x\right)-d^{\prime}(C d x) .
$$

The plane $Q S x$ will pass through $y$ if,

or,

$$
(Q S x y)=0,
$$

$$
\Omega\left(b b^{\prime}, d d^{\prime}\right) \cdot\left(x b^{\prime} d^{\prime} y\right)(x A b)(x C d)=0 .
$$

Simplifying by the usual identities, this is equivalent to

or,

$$
\Omega\left(d d^{\prime}\right) \cdot\left(x b^{\prime} b y\right)\left(x A d^{\prime}\right)(x C d)+\Omega(b d) \cdot\left(x b^{\prime} d^{\prime} b\right)(x A y)(x C d)
$$

$$
(x A y)(x B C D x)-(x B y)(x A C D x)=0,
$$

1 H. F. Baker, Principles of Geometry, 3, 170. 
which is the equation to the surface. This is the same form as obtained in $\$ 2$ (13) and (14), with $y$ a definite point depending on the six initial lines.

From the equation (1), or its equivalent,

$$
(x C y)(x D A B x)-(x D y)(x C A B x)=0,
$$

all the lines of the surface can be accounted for. In the Schläfli notation, $A B C D$ would be denoted by $a_{1} a_{2} a_{3} a_{4}$ while the two lines through $y$, one meeting $A$ and $B$, the other $C$ and $D$, would be $c_{12} c_{34}$. The $b_{5}$ and $b_{6}$ would be the pair of lines common to the two quadrics, other than $C$ and $D$,

$$
(x A C D x)=0=(x B C D x) .
$$

Also $b_{1}$ would be the line joining the two points in which the plane $(x B y)=0$ meets the lines $C$ and $D$ and similarly for the others. It is not my intention here to enter into the discussion of all the lines.

Again it may be verified without difficulty that (1) can be expressed in the form

$$
\begin{aligned}
(x A y) & \left(\Omega\left(d d^{\prime}\right) \cdot(C d x)\left(B y d^{\prime}\right)\right)\left(\Omega\left(a a^{\prime}\right) \cdot(B a x)\left(D a^{\prime} y\right)\right) \\
& +(x B y)\left(\Omega\left(d d^{\prime}\right) \cdot(C d x)\left(A y d^{\prime}\right)\right)\left(\Omega\left(b b^{\prime}\right) \cdot(A b x)\left(D b^{\prime} y\right)\right)=0
\end{aligned}
$$

each part being the product of three factors and expressed in a very symmetrical manner. This is of the well known type

$$
u v w+u^{\prime} v^{\prime} w^{\prime}=0
$$

where $u, v, \ldots, w^{\prime}$ are six planes.

A well known standard form ${ }^{1}$ for the surface which has 27 real and distinct lines is given by the equation

$\left(\frac{x}{x_{2}}+\frac{y}{y_{2}}+\frac{z}{z_{2}}+\frac{w}{w_{2}}\right)\left(\frac{x z}{x_{1} z_{1}}-\frac{y w}{y_{1} w_{1}}\right)=\left(\frac{x}{x_{1}}+\frac{y}{y_{1}}+\frac{z}{z_{1}}+\frac{w}{w_{1}}\right)\left(\frac{x z}{x_{2} z_{2}}-\frac{y w}{y_{2} w_{2}}\right)$.

It is found that the equation

$$
(x y A)(x B C D x)-(x y B)(x A C D x)=0
$$

can be transformed immediately into (4) by taking for the tetrahedron of reference two points on the line $C$ as $(0,0,0,1),(0,0,1,0)$, and

1 A. Henderson, The tuenty-seven lines of the Cubic Surface. Cambridge Tracts, 13 (1911). 
Equations to Cubic Surfade, with Extensions to Higher Space 9

two on the line $D$ as $(1,0,0,0),(0,1,0,0):$ the line $A$ as the join of $\left(x_{2}, 0,0,-t_{2}\right),\left(0, y_{2},-z_{2}, 0\right)$, and $B$ as the join of $\left(x_{1}, 0,0,-t_{1}\right)$, $\left(0, y_{1},-z_{1}, 0\right)$. The auxiliary point $y$ in this case has coordinates,

$\left\{\frac{\left(t_{1}-t_{2}\right) x_{1} x_{2}}{z_{1} z_{2} t_{1} t_{2}\left(x_{1} t_{2}-x_{2} t_{1}\right)}, \frac{\left(z_{1}-z_{2}\right) y_{1} y_{2}}{z_{1} z_{2} t_{1} t_{2}\left(y_{2} z_{1}-y_{1} z_{2}\right)}, \frac{\left(y_{1}-y_{2}\right)}{t_{1} t_{2}\left(y_{1} z_{2}-y_{2} z_{1}\right)}, \frac{\left(x_{1}-x_{2}\right)}{z_{1} z_{2}\left(x_{2} t_{1}-x_{1} t_{2}\right)}\right\}$.

The lines $C, D$ and the two transversals of $A B C D$ are the common generators of the two quadrics.

\section{$\S 4$.}

Extensions of the equation $\S 3(1)$ can be obtained and will be briefly illustrated for four and five dimensional space. These are not general, but the analytical form for the manifold in [5] is an exact extension to that for [3].

Consider the case in four space. Take a line and plane as being associated (analogous to the two lines in H. F. Baker's construction). Denote the line by $\left(a a^{\prime}\right)$ or $A_{2}$ and the plane (two-fold) by $\left(b b^{\prime} b^{\prime \prime}\right)$ or $B_{3}$, the five $a$ and $b$ symbols denoting points.

Take three pairs of this type and a given point $y$. Consider the quartic manifold built up in an analogous way to the Cubic Surface in [3]. Take a three-fold through $y$ to out the line $A_{2}$ in a point and the two-fold $B_{3}$ in a line with similar intersections for the other pairs: each point and line determines a plane in the three-fold space. These three planes will intersect in a point. The locus of this point for varying three-folds through $y$ will be a quartic manifold. Its equation is found to be

$$
\Omega\left(a a^{\prime}, c c^{\prime}, e e^{\prime}\right) \cdot\left(a B_{3} x\right)\left(c D_{3} x\right)\left(e F_{3} x\right)\left(a^{\prime} c^{\prime} e^{\prime} y x\right)=0 .
$$

The given two-folds are $B_{3}, D_{3}, F_{3}$, and the corresponding lines $A_{2}, C_{2}, E_{2}$.

The equation (4) does not admit of a symmetrical representation similar to $\S 3(1)$ but can be expressed as

$$
\begin{aligned}
\left(B_{3} y x\right) & \Omega\left(c c^{\prime}, e e^{\prime}\right) \cdot\left(D_{3} e x\right)\left(F_{3} e x\right)\left(A_{2} c^{\prime} e^{\prime} x\right) \\
& +\left(\Omega\left(c c^{\prime}\right) \cdot\left(B_{3} c^{\prime} x\right)\left(D_{3} c x\right)\right)\left(\Omega\left(e e^{\prime}\right) \cdot\left(F_{3} e x\right)\left(A_{2} e^{\prime} y x\right)\right) \\
& +\left(\Omega\left(e e^{\prime}\right) \cdot\left(B_{3} e^{\prime} x\right)\left(D_{3} e x\right)\right)\left(\Omega\left(c c^{\prime}\right) \cdot\left(D_{3} c x\right)\left(A_{2} c^{\prime} y x\right)\right)=0,
\end{aligned}
$$

which is of the type,

$$
a_{x} p_{x}^{3}+b_{x}^{2} c_{x}^{2}+d_{x}^{2} e_{x}^{2}=0
$$


The four quadric manifolds $b_{x}^{2}=0, c_{x}^{2}=0 \ldots$ are cones. Clearly the three two-folds, as well as the three lines, lie on the surface as well as the lines through the point $y$ intersecting the pairs $A_{2}, B_{3}$; $C_{2}, D_{3} ; E_{2}, F_{3}$; each two of the planes $B_{3}, D_{3}, F_{3}$ intersect in a point, and the three points so found determine a plane which meets the surface in a quartic curve which breaks up into four straight lines.

In the case of [5] space, six three-folds grouped in two threes $A_{4}, B_{4}, C_{4} ; D_{4}, E_{4}, F_{4}$ and an additional point $y$ are chosen: $A_{4}$ is the three-fold built up of the four points $a, a^{\prime}, a^{\prime \prime}, a^{\prime \prime \prime}$, with coordinates $\left(a a^{\prime} a^{\prime \prime} a^{\prime \prime \prime}\right)$.

Just as the Cubic Surface was obtained in $\S 3(1)$ as

$$
\Omega\left(b b^{\prime}, d d^{\prime}\right) \cdot\left(x A_{2} b\right)\left(x C_{2} d\right)\left(x b^{\prime} d^{\prime} y\right)=0,
$$

so, a quintic manifold in five has the same formation

$\Omega\left(c c^{\prime} c^{\prime \prime} c^{\prime \prime \prime}, f f^{\prime} f^{\prime \prime} f^{\prime \prime \prime}\right) .\left(x A_{4} c\right)\left(x B_{4} c^{\prime}\right)\left(x D_{4} f\right)\left(x E_{4} f^{\prime}\right)\left(x c^{\prime \prime} c^{\prime \prime} f^{\prime \prime} f^{\prime \prime \prime} y\right)=0$,

where $\Omega$ is a determinantal permutation of $c, c^{\prime}, c^{\prime \prime}, c^{\prime \prime \prime}$, and an independent one of $f, f^{\prime}, f^{\prime \prime}, f^{\prime \prime \prime}$.

Take the first and last bracket factors in (5), and it reduces to

$$
\begin{aligned}
& \left(x A_{4} y\right) \Omega(c \ldots, f \ldots) \cdot\left(x B_{4} c^{\prime}\right)\left(x D_{4} f\right)\left(x E_{4} f^{\prime}\right)\left(x c^{\prime \prime} c^{\prime \prime \prime} f^{\prime \prime} f^{\prime \prime \prime} c\right) \\
& \quad+\Omega(c \ldots, f \ldots) \cdot\left(x A_{4} f^{\prime \prime}\right)\left(x B_{4} c^{\prime}\right)\left(x D_{4} f\right)\left(x E_{4} f^{\prime}\right)\left(x c^{\prime \prime} c^{\prime \prime \prime} c f^{\prime \prime \prime} y\right)=0
\end{aligned}
$$

or

$$
\begin{aligned}
\Omega(f \ldots) & \left(\left(x A_{4} y\right)\left(x D_{4} f\right)\left(x E_{4} f^{\prime}\right)\left(x B_{4} f^{\prime \prime}\right)\left(x C_{4} f^{\prime \prime \prime}\right)\right. \\
& +\left(x B_{4} y\right)\left(x D_{4} f\right)\left(x E_{4} f^{\prime}\right)\left(x C_{4} f^{\prime \prime}\right)\left(x A_{4} f^{\prime \prime \prime}\right) \\
& \left.+\left(x C_{4} y\right)\left(x D_{4} f\right)\left(x E_{4} f^{\prime}\right)\left(x A_{4} f^{\prime \prime}\right)\left(x B_{4} f^{\prime \prime \prime}\right)\right)=0 .
\end{aligned}
$$

This is capable of an alternative expression with the rôles of $A_{4} B_{4} C_{4}$, and $D_{4} E_{4} F_{4}$ interchanged.

It is at once clear that the six planes $A_{4}, \ldots$, all lie on the quintic manifold in addition to the plane common to

$$
\left(x A_{4} y\right)=0=\left(x B_{4} y\right)=\left(x C_{4} y\right),
$$

and that common to

$$
\left(x C_{4} y\right)=0=\left(x D_{4} y\right)=\left(x E_{4} y\right) .
$$


Equations to Cubic Surface, ifith Extensions to Higher Space 11 This is analogous to the property of the Cubic Surface

$$
(x A y)(x B C D x)-(x B y)(x A C D x)=0,
$$

where a line through $y$ which meets $A$ and $B$, namely that common to $(x A y)=0=(x B y)$, lies on the surface.

Again the quartic manifold, $\S 1(8)$,

$$
\Omega(f \ldots) \cdot\left(x D_{4} f\right)\left(x E_{4} f^{\prime}\right)\left(x B_{4} f^{\prime \prime}\right)\left(x C_{4} f^{\prime \prime \prime}\right)=0
$$

is formed by exactly the same method as the quadric surface

in [3].

$$
(x B C D x)=0
$$

